\title{
The research on post processing algorithm of B-C five-axis
}

\author{
Qingting $\mathrm{Li}^{1,}$, , BingYan ${ }^{2}$, Wei Zhao ${ }^{2}$ \\ ${ }^{1}$ Tianjin University of technology and education, Tianjin 300222, China \\ ${ }^{2}$ Graduated from Tianjin University, Tianjin 300222, China \\ a398393146@qq.com
}

Keywords: Five coordinates; post processing; Tool path; the interface conversion.

\begin{abstract}
According to the structure and motion transformation characteristics of five coordinate of machine tool through the establishment of mathematical model, get the coordinate transformation formula of rear processing, tool path, by using CATIA and other CAM software, tool path converted to machine tool able to identify the NC code based on the Delphi language compiled interface conversion .Through simulation verification in the verdict, validate the correctness of the post processing algorithm program, for the actual processing demand, has important application value.
\end{abstract}

\section{Foreword}

Multi-axis machining means increasing the rotating axis which is based on the traditional three axis CNC machine. Five-axis linkage CNC machine tools means increasing around the $\mathrm{X}, \mathrm{Y}, \mathrm{Z}$ any two axis of rotation which is based on the $\mathrm{X}, \mathrm{Y}, \mathrm{Z}$ axis, the linear motion of the three directions .Five-axis machine tool in machining process, is to assume that the workpiece stationary, and rotation of the cutting tool. Because of five-axis linkage machine tool structure is varied, mainly includes: five coordinate linkage double rotating machine tools, five coordinate linkage double pendulum head and five coordinates linkage head and turn the machine tool ${ }^{[1]}$. Method of processing is also very flexible, and also puts forward very high requirements to the operator. In real life, for machining complex parts, directly programming, typically used in nc machining, but this approach has a high requirement to the operators. This article mainly through the CAM software such as CATIA obtain tool path, based on Delphi platform, converted into NC code, and then the simulation verification in vericut, without interference, collision errors, used for the actual machine tool processing. This article is mainly aimed at B - C "tool swinging and rotation table" five coordinate motion structure, in view of the DM G company DMC75VLinear five-axis high speed machining center, CNC system is Heidenhain iTNC530 system ${ }^{[2]}$.Structure is as follows.

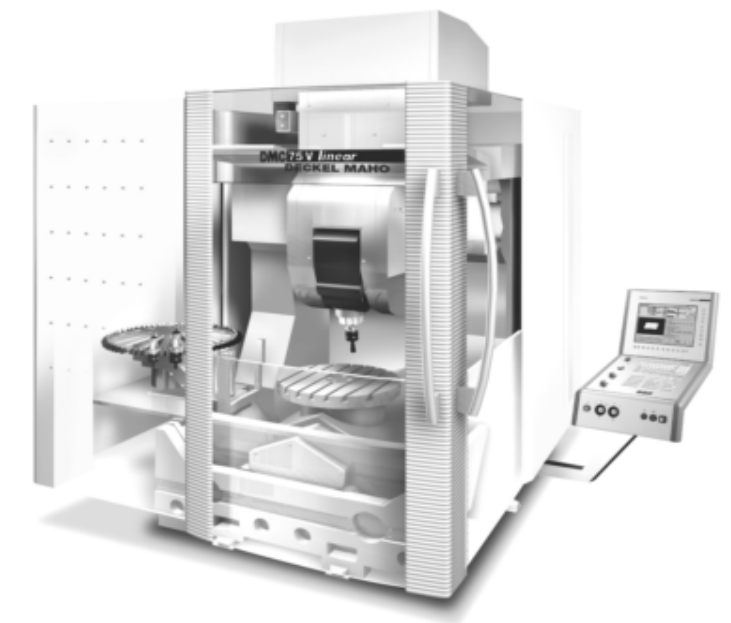

Figure 1. The structure of DMC75VLinear five-axis high speed machining center 
The main functions of Post processing: first, describe the tool motion path including the tip point and cutter axis vectors, converts it to the NC instruction data which five-axis machine tool able to identify. According to the C-B machine's motion transformation characteristics by using its structural characteristics, through the establishment of mathematical model enables data transfer and exchange uses between machine tool coordinate system and the workpiece coordinate system, due to the variable of movement structure of the machine, the transformation result is not the same ${ }^{[3-5]}$.

\section{The transformation of machine coordinate and solving the $x, y, z, c, b$}

\subsection{Establish a five-axis linkage machine coordinate system}

Generally regarded OwXwYwZw as workpiece coordinate system, OcXcYcZc as tool coordinate system .Post processing's main function is to convert cutting tool motion trajectory data in the workpiece coordinate system into the values in the machine tool coordinate system ${ }^{[6]}$.Due to get the tool path describes cutter location point and the tool axis vector based on workpiece coordinate system .Coordinate transformation is theoretically cutter location point and tool axis vector of the workpiece coordinate system transformed into the machine tool coordinate system, the basis of generating NC of five coordinate linkage machining. The actual calculation process, a rebellious thoughts, convert cutter location point and tool axis vector into the workpiece coordinate system. Figure 1 gives the motion artifacts geometry model of five coordinate machine

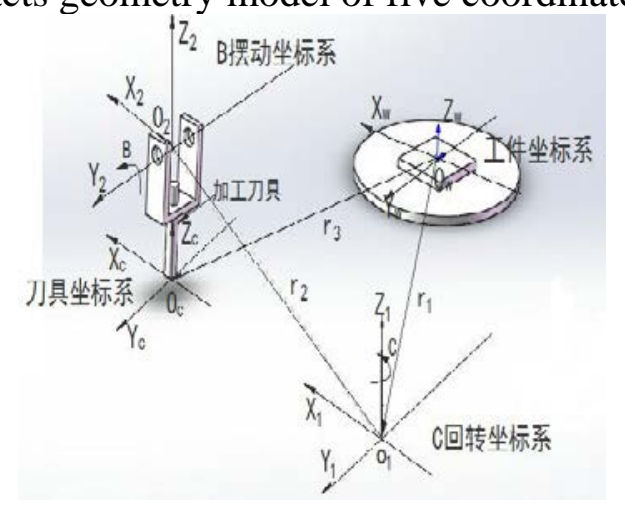

Figure 1.geometry model of motion artifacts in five coordinate machine

\subsection{Setting up five coordinate nc machine which is based on the mathematical model}

Initial state: Makes the tool axis and $\mathrm{Z}$ axis parallel; Assume that the distance from $\mathrm{O}_{\mathrm{C}}$ to $\mathrm{O}_{2}$, That is to say, $\mathrm{O}_{2} \mathrm{O}_{C}$ 's length is $\mathrm{L}$ (determined by the pendulum shaft length and the cutting tool length); the origin $\mathrm{O}_{2}$ of swing coordinate system $\mathrm{O}_{2} \mathrm{X}_{2} \mathrm{Y}_{2} \mathrm{Z}_{2}$, which position in the tool coordinate system is $\mathrm{r}_{2}$ $(0,0, L)$. The origin $\mathrm{O}_{1}$ of rotating coordinate system $\mathrm{O}_{1} \mathrm{X}_{1} \mathrm{Y}_{1} \mathrm{Z}_{1}$, which position in the workpiece coordinate system is the $r_{1}(m x, m y, m z)$.By defining the five coordinate machine processing, the initial state in the translational along a straight line axis X, Y, Z displacement vector for rs (sx, sy, sz), namely X,Y,Z coordinate values in the NC . Rotating shaft C, B of rotation Angle relative to the initial conditions forc, b ,namely C, B Angle value in the machine tool NC program .B angle rotation range is: $110^{\circ}$ to $11^{\circ}$, c angle rotation range is: $-360^{\circ}$ to $360^{\circ}$. Matrix transformation is as follows: The position of the workpiece coordinate system translational transformation $\mathrm{T}$ (r1) is relative to rotary axis:

$$
T\left(r_{1}\right)=\left[\begin{array}{llll}
1 & 0 & 0 & m_{x} \\
0 & 1 & 0 & m_{y} \\
0 & 0 & 1 & m_{z} \\
0 & 0 & 0 & 1
\end{array}\right]
$$

Tool endpoint relative to the translation of the rotary center tool matrix transformation, namely OcXcYcZc relative to $\mathrm{O}_{2} \mathrm{X}_{2} \mathrm{Y}_{2} \mathrm{Z}_{2}$ translation matrix transformation $\mathrm{T}(\mathrm{r} 2)$ :

$$
T\left(r_{2}\right)=\left[\begin{array}{llll}
1 & 0 & 0 & 0 \\
0 & 1 & 0 & 0 \\
0 & 0 & 1 & L \\
0 & 0 & 0 & 1
\end{array}\right]
$$


Rotary table relative to the workpiece rotation matrix transformation, namely $\mathrm{O}_{1} \mathrm{X}_{1} \mathrm{Y}_{1} \mathrm{Z}_{1}$ relative to OwXwYwZw rotating coordinate system, the actual movement direction of the workbench is contrary to the movement direction of the cutting tool relative to the workpiece for $T\left(r_{s}-r_{1}+r_{2}\right)$

$$
T\left(r_{s}-r_{1}+r_{2}\right)=\left[\begin{array}{llll}
1 & 0 & 0 & S_{x}-\boldsymbol{m}_{x} \\
0 & 1 & 0 & S_{y}-\boldsymbol{m}_{y} \\
0 & 0 & 1 & S_{z}-\boldsymbol{m}_{z}+L \\
0 & 0 & 0 & 1
\end{array}\right]
$$

Workpiece rotation transformation and transformation of rotary table corresponding to the matrix transformation relations, namely the rotation of the coordinate system transformation between $\mathrm{O}_{1} \mathrm{X}_{1} \mathrm{Y}_{1} \mathrm{Z}_{1}$ and $\mathrm{OwXwYwZw}$. In the axial direction of the workpiece, tool instead in the workbench (artifacts) of actual movement direction. The cutting tool endpoint relative to the rotation of the rotary center of tools matrix transformation, namely OcXcYcZc relative to $\mathrm{O}_{2} \mathrm{X}_{2} \mathrm{Y}_{2} \mathrm{Z}_{2}$ rotation matrix $R_{Y}(\mathrm{~b})$.

$$
\begin{aligned}
& \boldsymbol{R}_{Z}(-c)=\left[\begin{array}{llll}
\cos c & \sin c & 0 & 0 \\
-\sin c & \cos c & 0 & 0 \\
0 & 0 & 1 & 0 \\
0 & 0 & 0 & 1
\end{array}\right] \\
& R_{Y}(\mathrm{~b})=\left[\begin{array}{llll}
\cos b & 0 & \sin b & 0 \\
0 & 1 & 0 & 0 \\
-\sin b & 0 & \cos b & 0 \\
0 & 0 & 0 & 1
\end{array}\right]
\end{aligned}
$$

\subsection{The resolution of $x, y, z, c, b$ about five coordinate $n$ c machine tools.}

First get their cutter location point and cutter axis vector, these vectors in the tool coordinate system, respectively $\left[\begin{array}{lll}0 & 0 & 0\end{array}\right]^{T}\left[\begin{array}{lll}0 & 0 & 1\end{array}\right]^{T}$.cutter location point and cutter axis vector in the workpiece coordinate system it respectively $r_{u}\left(\begin{array}{llll}u_{x} & u_{y} & u_{z}\end{array}\right) r_{p}\left(\begin{array}{lll}p_{x} & p_{y} & p_{z}\end{array}\right)$, and also is the CLFS output file GOTO point location (x, y, z, i, j, k).The motion process of machine tool by:

(1) The rotation of OcXcYcZc relative to O2X2Y2Z2

translation O2X2Y2Z2 relative to O1X1Y1Z1

(3)The rotation of O1X1Y1Z1 relative to OwXwYwZw, can get the rs (sx, sy, sz), c, b.Get five coordinate machine kinematic chain coordinate transformation formula by matrix transformation relationship:

$$
\left[\begin{array}{llll}
u_{x} & u_{y} & u_{z} & 0
\end{array}\right]^{T}=T\left(r_{1}\right) * R_{z}(-c) * T\left(r_{s}-r_{1}+r_{2}\right) * R_{r}(b) * T\left(-r_{2}\right) *\left[\begin{array}{llll}
0 & 0 & 1 & 0
\end{array}\right]^{T}
$$

The formula (1)-(5) into the formula (6) is calculated:

$$
\left[\begin{array}{c}
u_{x} \\
u_{y} \\
u_{z} \\
0
\end{array}\right]=\left[\begin{array}{c}
\cos C^{*} \sin b \\
-\sin C^{*} \sin b \\
\cos b \\
0
\end{array}\right]
$$

cutter location position vector:

$$
\left[\begin{array}{llll}
p_{x} & p_{y} & p_{z} & 1
\end{array}\right]^{T}=T\left(r_{1}\right) * R_{z}(-c) * T\left(r_{s}-r_{1}+r_{2}\right) * R_{Y}(\mathrm{~b}) * T\left(-r_{2}\right) *\left[\begin{array}{llll}
0 & 0 & 1 & 0
\end{array}\right]^{T}
$$

The formula (1)-(5) into the formula (8) is calculated:

$$
\left[\begin{array}{c}
p_{x} \\
p_{y} \\
p_{z} \\
1
\end{array}\right]=\left[\begin{array}{c}
-\cos c^{*} \sin b^{*} L+\cos c^{*}\left(\boldsymbol{S}_{x}-\boldsymbol{m}_{x}\right)+\sin c^{*}\left(\boldsymbol{S}_{y}-\boldsymbol{m}_{y}\right)+\boldsymbol{m}_{x} \\
\sin c^{*} \sin b^{*} L-\sin c^{*}\left(\boldsymbol{S}_{x}-\boldsymbol{M}_{x}\right)+\cos c^{*}\left(\boldsymbol{S}_{y}-\boldsymbol{m}_{y}\right)+\boldsymbol{m}_{y} \\
-\cos b^{*} L+L \\
1
\end{array}\right]
$$


The axis displacement at vector at any time in the machine tool movement can be obtained by simultaneous equation (7) (9) :

$$
b=\arccos u_{z} \quad c=-\arctan \left(u_{y} / u_{x}\right)
$$

By the formula (7) :

$$
u_{x}{ }^{2}+u_{y}{ }^{2}=\sin ^{2} b \quad \frac{u_{z}}{\sqrt{u_{x}{ }^{2}+u_{y}{ }^{2}}}=\frac{\cos b}{|\sin b|}= \pm \cot b
$$

As a result of $b$ in $(110,90),(90,0)$, sinb less than zero, namely

$$
\frac{u_{z}}{\sqrt{u_{x}{ }^{2}+u_{y}^{2}}}=-\cot b \quad b=-\cot ^{-1} \frac{u_{z}}{\sqrt{u_{x}^{2}{ }^{+} u^{2}}} \quad b=\cot ^{-1} \frac{u_{z}}{\sqrt{u_{x}^{2}{ }^{+} u_{y}^{2}}}
$$

When the $\mathrm{b}$ in $(0,11)$ :By the formula $(10)$,in the post-processing algorithm decided to $\mathrm{c}$ rotation angle of the positive and negative judgment and the cutter axis vector ux, uy.So CLSF files,in pre-processing $\mathrm{u}_{\mathrm{x}}, \mathrm{u}_{\mathrm{y}}$ will be the plus and minus sign or zero, namely the ux, uy will be taking the $\mathrm{X}, \mathrm{Y}$ plane is divided into four quadrants to discuss, to determine the $\mathrm{c}$ angle rotation and direction of $\mathrm{c}$ angle .Discuss the classification:

(1)the $u_{x}$ is not equal to zero, $u_{y}$ is equal to zero, it is concluded that $\mathrm{c}=0$;

(2) the $u_{x}$ is equal to zero, $u_{y}$ is not equal to zero, $c=-90\left(u_{y} .>0\right)$; $C=90\left(u_{y} \cdot<0\right)$

(3) the $\mathrm{u}_{\mathrm{x}}$ is not equal to zero, $\mathrm{u}_{\mathrm{y}}$ is not equal to zero, ${ }_{c=-\arctan (}\left(u_{\mathrm{y}} / u_{x}\right)+180 * \mathrm{~K}(\mathrm{~K}=-1,1)$

Translation coordinates are obtained by formula (9):

$$
\begin{aligned}
& \boldsymbol{S}_{x}=\left(\boldsymbol{p}_{x}-\boldsymbol{m}_{x}\right) * \operatorname{cosc}-\left(p_{y}-\boldsymbol{m}_{y}\right) * \operatorname{sinc}+2 \operatorname{sinb} * \mathrm{~L}+\boldsymbol{m}_{x} \\
& \boldsymbol{S}_{y}=\left(\boldsymbol{p}_{x}-\boldsymbol{m}_{x}\right) * \operatorname{sinc}+\left(\boldsymbol{p}_{y}-\boldsymbol{m}_{y}\right) * \operatorname{cosc}+\boldsymbol{m}_{y} \\
& \boldsymbol{S}_{z}=\boldsymbol{p}_{z}-(1-\operatorname{cosb}) * L
\end{aligned}
$$

Suppose the initial state when the tool origin and workpiece origin, can get $\mathrm{mx}=0$, my $=0$, into (10) to sort out are:

$$
\begin{aligned}
& S_{x}=p_{x}^{*} \cos c-p_{y}^{*} \operatorname{sinc}+2 \sin b^{*} \mathrm{~L} \\
& S_{y}=p_{x}^{*} \operatorname{sinc}+p_{y}^{*} \cos c \\
& S_{z}=p_{z}-(1-\cos b)^{*} L
\end{aligned}
$$

\section{The implementation and validation of post processing}

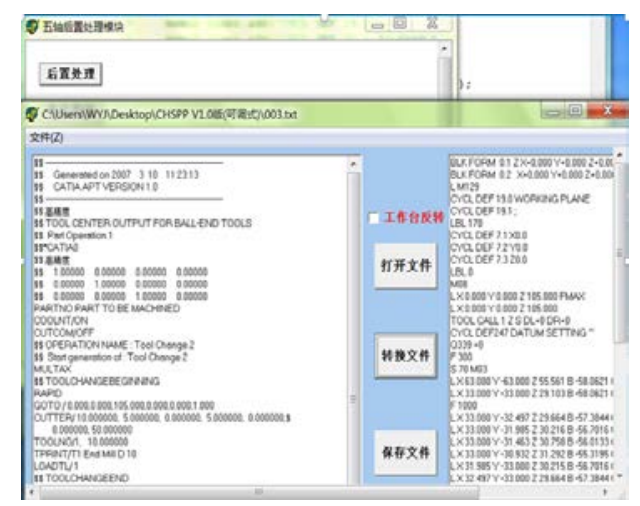

Figure 3.conversion interface 
File. Using CATIA parts with complex curved surface model, and then, after a series of nc machining setting for an APT cutter location file.

Parts model by using CATIA software, the generated APT file, transformed into NC based on Delphi platform.

DMC75VLinear five-axis machine imported into the vericut as shown

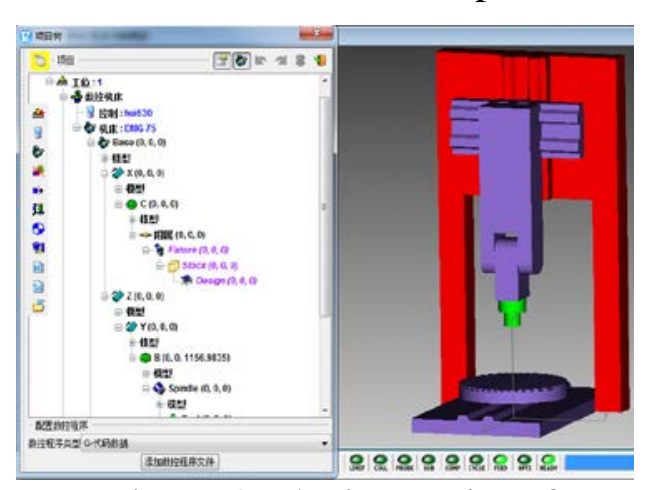

Figure 4. The import interface

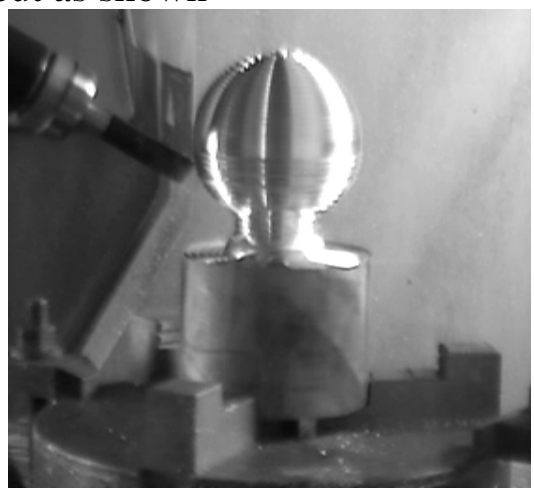

Figure 5. The simulation process

Processing simulation in the virtual machine tool

\section{Summary}

Five-axis nc machining becomes a trend of current CNC numerical control processing technology, can be used for machining complex curved surface parts, relative to the 3 axis nc machine tools, can improve the efficiency of the mechanic, the quality of machining parts. But because of the diversity of the five coordinate machine, post processing software given by manufacturer when processing tend to go wrong, so research and development special post processing program has become the current urgent task. According to the structure of B - C machine and kinematic transformation, through post processing algorithm, get coordinates formula, And verified the correctness of the algorith.

\section{References}

[1].Wang Gang, Zhao Wan-sheng, Shi Xuming. Numerical control machine tool general post processing program development [J]. Mechanical, 2007, 27 (3).

[2].Tang Zhen-yu. Linear motor feed drive technology in the application of n c machine tool [J]. Journal of machine tools and hydraulic, 20095 (3) : 67-70

[3].Jin Rong. Five coordinate linkage CNC technology [M]. Changsha: hu nan science and technology press, 1995.

[4].Pang Jiwei. Rear five-axis nc milling processing and processing programming research [D]. Jinan: shandong university, 2007.

[5].Ai-ling wang, meng-qun li, Feng Yujiang. Nc machining theory and practical technology [M]. Beijing: mechanical industry publishing house, 2009.

[6].Liu Riliang, Cheng Ruizhang, Song Xianchun and so on . A special case of 5 axis nc machine tool coordinate system and its post processing method. The mechanical design and manufacturing engineering, 20. 